\title{
Low-Loss Simple Waveguide Intersection in Sillicon Photonics
}

\author{
Fumihiro Shinobu, Yoshiki Arita and Toshihiko Baba
}

We propose a simple silicon wire intersection with low diffraction loss and crosstalk. Finite-difference time-domain analysis shows the lowest loss to be as small as $0.14 \mathrm{~dB}$. We fabricated an optimum structure on SOI substrate and measured the loss to be $0.12 \mathrm{~dB}$, which might be reduced by the smoothing of the fabricated structure.

For high-density photonic integrated circuits, low-loss and low-crosstalk intersection of waveguides is a basic but important component. However, in highindex-contrast waveguides such as photonic wires in Si photonics, a $1.2 \mathrm{~dB}$ loss and $-11.5 \mathrm{~dB}$ crosstalk occur even in a simple intersection shown in Fig. 1(a) due to strong diffraction of the guided mode. The diffraction can be suppressed by expanding the guided mode in a taper, as shown Fig. 1(b). We have shown using three-dimensional finite-difference time-domain (FDTD) analysis that the loss can be reduced to $0.4 \mathrm{~dB}$ by employing an elliptical taper structure [1]. However, this value is not sufficiently low for high-density interconnects containing many intersections. Several similar structures have been discussed and demonstrated [2-4]. In particular, tapers fabricated by a two-step etching process smoothens the mode expansion and reduces the loss to $0.14 \mathrm{~dB}$ [4]. In this paper, we propose the intersection shown in Fig. 1(c), which can be fabricated by a single-step process, and completely suppresses diffraction by slightly modulating the standard taper structure (b). In the standard structure, sidewalls of two waveguides cross at $\geq 90^{\circ}$, in which case diffraction loss is reduced but cannot be suppressed completely at the center of the intersection. The proposed structure (c) consists of four offset ellipses. Since the taper is slightly narrowed just before the center of the intersection, light is focused toward the center and diffraction is suppressed.

First, we performed an FDTD analysis of the mode propagation through a structure corresponding to the fabricated device described below; Si (index $n=3.5$ ) wire of $400 \mathrm{~nm}$ width and $220 \mathrm{~nm}$ thickness cladded by $\mathrm{SiO}_{2}(n=1.45)$. The majoraxis $2 a$, minor-axis $2 b$, and offset-length $x$ were varied as parameters. The wavelength was set at $\lambda=1.55 \mu \mathrm{m}$, and the polarization fixed to transverse electric (TE). Fig. 1 shows calculated profiles of light propagation (magnetic field normal to the plane). In the simple intersection (a), diffraction is clearly observed. In the standard taper (b), the profile asymmetry between the left and right hand side suggests a small amount of diffraction. In the proposed structure (c), the mode is first expanded in the ellipse, then focused slowly so that the focal point is located at the center of the intersection, and finally transmitted to the opposite side. The profile is almost symmetric with the minimum loss evaluated to be $0.14 \mathrm{~dB}$ for $a=3.60 \mu \mathrm{m}, b=$ $1.00 \mu \mathrm{m}$ and $x=5.36 \mu \mathrm{m}$.

We fabricated the device on an SOI substrate using the foundry service NTTATN, where the pattern is formed by electron beam direct writing. Many intersections were inserted with a $50 \mu \mathrm{m}$ period into the photonic wire waveguide with the same design parameters as in the FDTD analysis. The number of intersections $N$ was set at $5,10,20$ and 30 . Both ends of the waveguide were terminated by spot-size converters consisting of inverse tapers and $\mathrm{SiON} / \mathrm{SiO}_{2}$ low-index-contrast large core waveguides. For the intersection, we fixed $a+x=12.06 \mu \mathrm{m}$ and $b=1.19 \mu \mathrm{m}$, and changed $a$ in each sample. The value of $a+x$ is slightly different from the above optimum value, because we fabricated the device before completing the optimization. In the measurement, light from a tunable laser source was coupled to the spot-size converter using objective lenses in TE polarization. The output light was collected by another objective lens and measured by optical power meter. Fig. 2 shows transmission spectra of samples with different $N$. It exhibits the broad band 
transmission characteristics at $\lambda=1.50-1.63 \mu \mathrm{m}$. Fine oscillation of $<1 \mathrm{~dB}$ amplitude in the spectra is caused by the reflection of waveguide end facets. On the other hand, slow oscillation is observed clearly for $N \geq 20$. We believe this is due to Fabry-Perot resonance arising from the starting and ending points of the intersection. From the period of oscillation, we infer the cavity length to be $13 \mu \mathrm{m}$ for a group index $n_{\mathrm{g}}=4.5$ [5], which is equivalent to the length of the intersection. Furthermore, the insert in Fig. 2 shows the combined near-field pattern / optical micrograph of the device, showing scattering at the device end points and further supporting our hypothesis. Fig. 3 summarizes the transmission intensity with varying $N$ and the loss per intersection at $\lambda=1.55 \mu \mathrm{m}$. The intensity decreases monotonically with $N$, suggesting each intersection gives a uniform loss. The experimental and numerical results are in good agreement, indicating the minimum loss to occur at $a=5.67 \mu \mathrm{m}$. The average loss is determined to be $0.24 \mathrm{~dB}$, which is almost the same as the theoretical value of $0.23 \mathrm{~dB}$.

We fabricated the optimally designed structure by using foundry service of IME, where the pattern is formed by optical lithography. Then, we evaluated the lowest loss of $0.12 \mathrm{~dB}$ in the same way as described above. This value is smaller than the calculated value. We noticed through the FDTD calculation that slight smoothing of the device end points can reduce the theoretical loss to less than $0.1 \mathrm{~dB}$. The experimental value might be due to such smoothing which occurs in the optical lithography with a relatively low resolution. This is the lowest loss of Si wire intersection demonstrated to date. This work was partly supported by the JSPS FIRST Program.

\section{References}

1 Fukazawa, T., Ohno, F., and Baba, T.: 'Very compact arrayed-waveguide-grating demultiplexer using Si photonic wire waveguides', Jpn J. Appl. Phys., 2004, 43, (2), pp. L673 - L675

2 Chen, H., and Poon, A. W.: 'Low-loss multimode-interference-based crossings for silicon wire waveguides', IEEE Photon. Technol. Lett., 2006, 18, (21), pp. 22602262

3 Zhu, Z., Wang, Z., Chang, S.J., and Chen, Y. J.: 'A novel approach to reduce waveguide intersection loss of HIC materials',OFC/NFOEC , 2007, paper JWG25

4 Bogaerts, W., Dumon, P., and Thourhout, D. V.; 'Low-loss, low-cross-talk crossings for silicon-on-insulator nanophotonic waveguides', Opt. Lett., 2007, 32 , (19), pp. 2801-2803

5 Sakai, A., Hara, G., and Baba, T.: 'Propagation characteristics of ultra-high $\Delta$ optical waveguide on silicon-on-insulator substrate', Jpn. J. Appl. Phys., 2002, 38, (7), pp. 743-752

\section{Authors' affiliations:}

F. Shinobu, Y. Arita and T. Baba (Department of Electrical and Computer Engineering, Yokohama National University, 79-5, Tokiwadai, Hodogaya, Yokohama, Kanagawa, 241-8501, Japan. They also belong to CREST, Japan Science and Technology Agency, Japan.)

E-mail: baba@ynu.ac.jp 


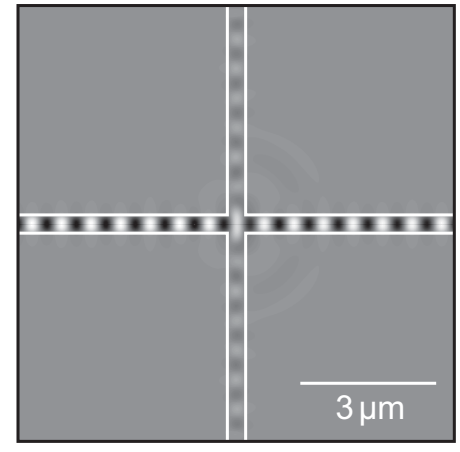

(a)
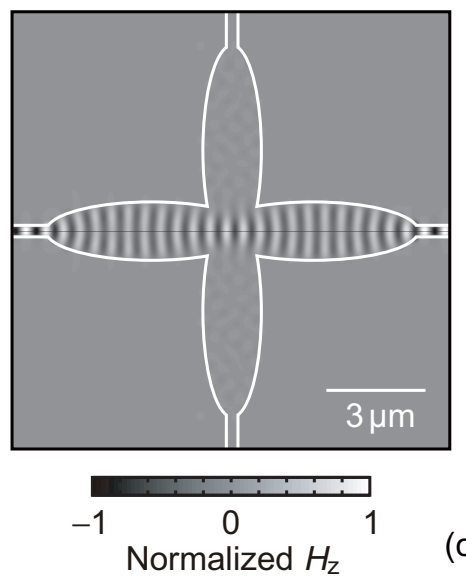

(c)

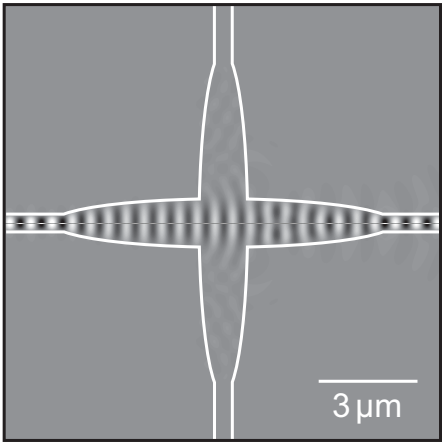

(b)

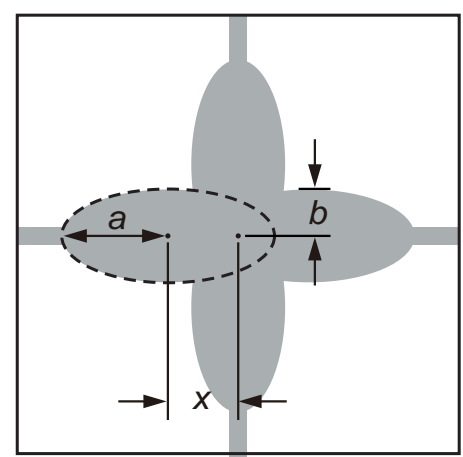

Fig. 1 Structure of intersection and calculated modal profile. (a) Simple crossstructure. (b) Standard taper structure. $a=5.00 \mu \mathrm{m}$. $b=0.80 \mu \mathrm{m}$. (c) Proposed structure. $a=5.00 \mu \mathrm{m} . b=0.80 \mu \mathrm{m} . x=1.76 \mu \mathrm{m}$. Right figure shows parameters.

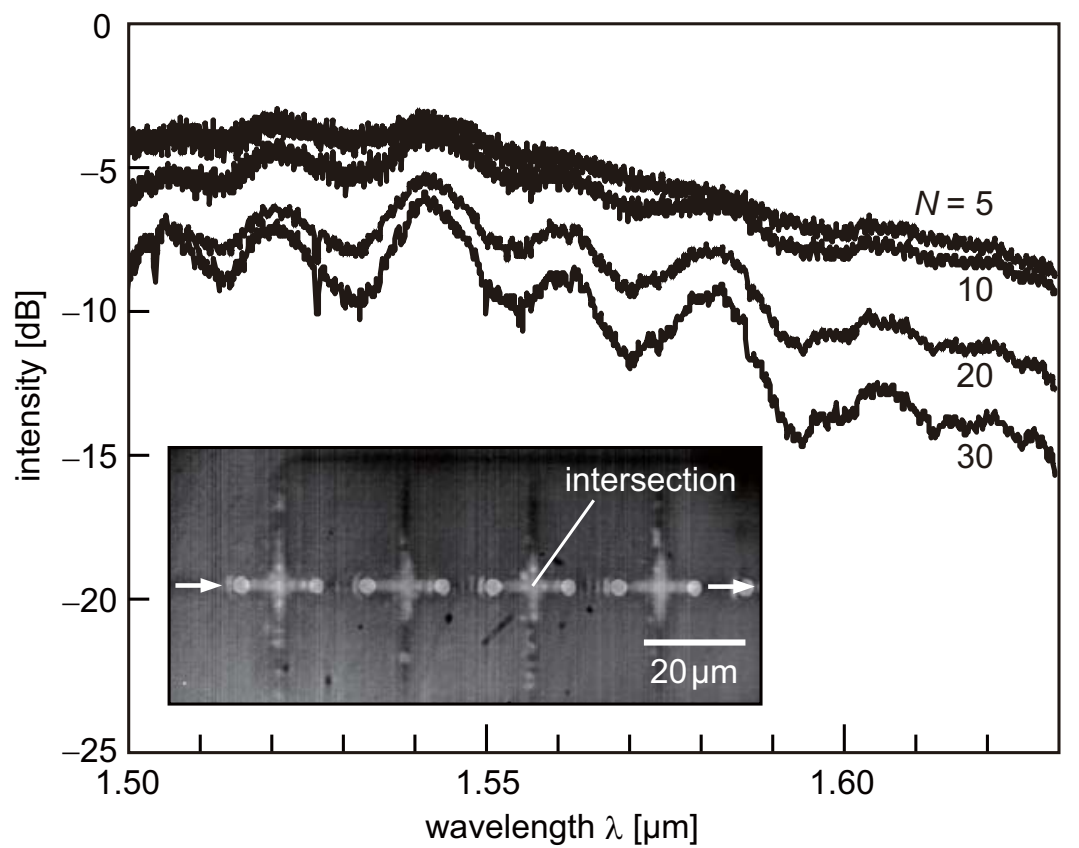

Fig. 2 Transmission spectra of fabricated intersections $(a=5.76 \mu \mathrm{m})$. Intensity is normalized by that without sample. Inset shows the near-field pattern of scattered light at $\lambda=1.55 \mu \mathrm{m}$ overlapped with optical microscope image of the device. 


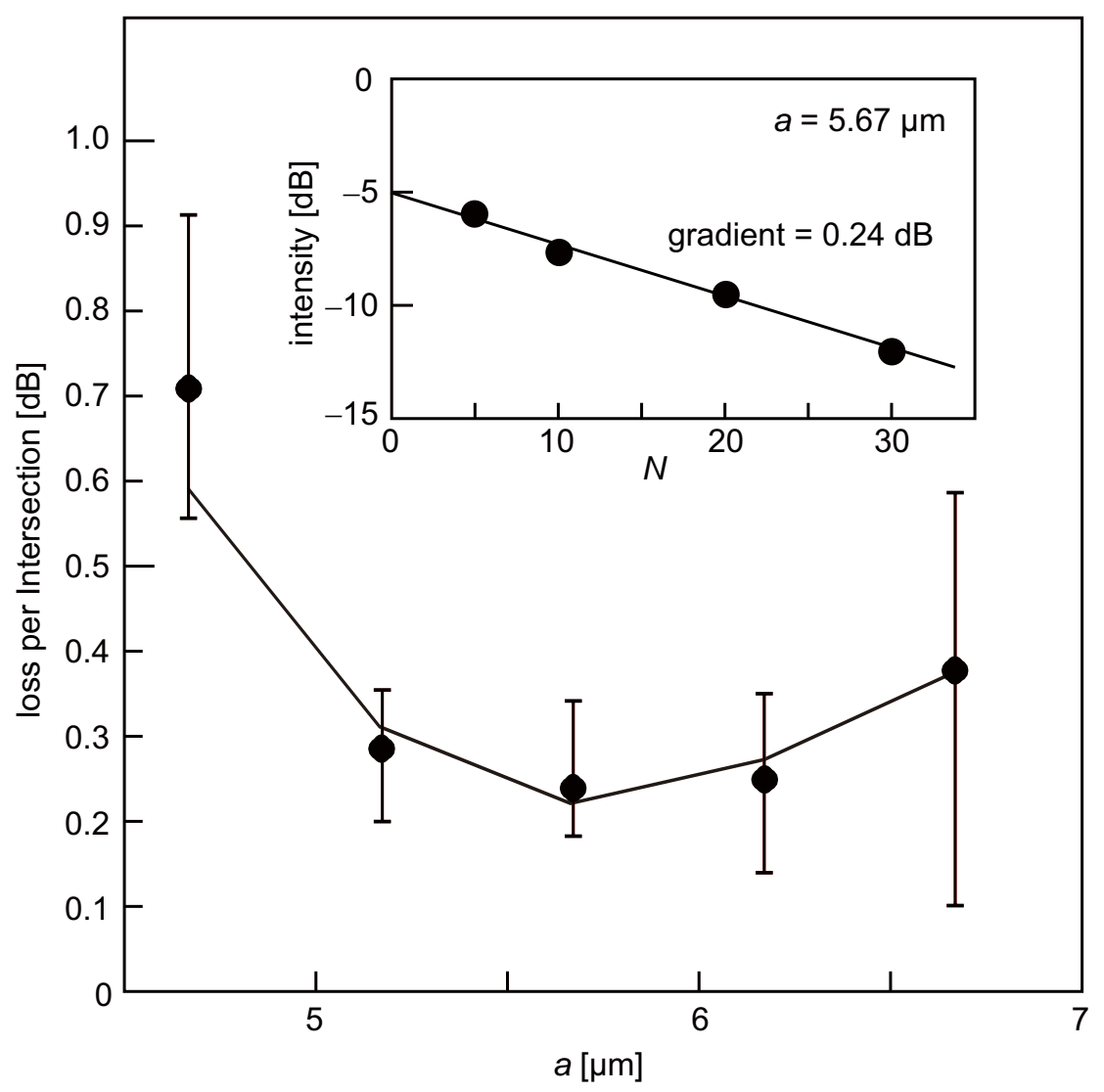

Fig. 3 Loss per intersection at $\lambda=1.55 \mu \mathrm{m}$. Solid line shows FDTD simulation, and circular plots, the average values of measured results. Inset shows the change in output intensity with $N$ at $a=5.67 \mu \mathrm{m}$. 